\title{
3D CAPABILITIES OF PLEIADES SATELLITE
}

\author{
M. Bernard, D. Decluseau, L.Gabet, P. Nonin, \\ ASTRIUM GEO-Information Services - (Marc.Bernard, David.Decluseau, Laurent.Gabet, \\ Philippe.Nonin)@ spotimage.fr
}

KEY WORDS: Photogrammetry, Automation, Matching, DEM/DTM, Satellite, Accuracy, Stereoscopic

\begin{abstract}
:
End of 2011 a new optical satellite, called Pléiades, was launched by the French space agency (CNES). It provides 20kmx20km images at 0.5 meters. This agile acquisition system is able to relocate very rapidly and scan the earth in any direction. The agility of the system offers the ability to acquire multi viewing angle images of the same area during the same orbit. This ability to capture, from a single stereoscopic pair, to a sequence of 25 images, allows enhancing the quality and the completeness of automatically extracted 3D maps.

The aim of the study is to validate and quantify the capacity of the Pléiades system to perform 3D mapping. The analysis explores the advantages in terms of quality and automatism to use more than 2 stereoscopic images.

In the last 10 years, automatic 3D processing of digital images became more and more popular and efficient. Thanks to aerial images with very large overlap and very high resolution satellite images, new methodologies and algorithms have been implemented to improve the quality and accuracy of automatic 3D processing. We propose to experiment the same type of approaches using Pléiades images to produce digital elevation models (DEM). A focus is made on analysing the 3D processing using video like (multi viewing) acquisitions. Different reference sites with very accurate 3D control points are used to quantify the quality of the Pléiades DEM. Different acquisition modes are explored from a single stereo pair to a sequence of 17 images.
\end{abstract}

\section{INTRODUCTION}

The agility of the new generation of earth observation satellites aims at increasing the acquisition capacity, allowing nearby areas to be acquired during the same pass. A side effect is the ability to acquire multiple views from the same area, with different incidence angles. Using multiple views for 3D automatic extraction is a common practice in the airborne survey community. The main motivations are the improvement of the robustness and accuracy, due to the redundancy of the measurements, and the removal of any hidden part in urban area, due to the multiplicity of the viewing angles.

Based on these considerations, the applicability of airborne multiview techniques to agile satellites imagery is a worthwhile question. This paper analyses the performances of this approach with Pleiades imagery.

\section{BACKGROUND}

The automatic matching of images stereo pairs has been widely used for more than thirty years in the mapping industry for the production of digital elevation models (DEM). For airborne frame cameras, the usual acquisition scheme is based on $60 \%$ overlaps in the flight direction with a minimal overlap between adjacent tracks. This scheme insures that every point on the ground is seen from, at least, two different points of view, which seems to be sufficient for an open landscape, with moderate slopes and a manual digitizing process. In an urban environment, more points of view are required to ensure the completeness of the elevation model, all around the highest buildings. Moreover, the redundancy of the stereo measurements from multiple points of view improves the robustness of an automatic images matching process. In any cases, the accuracy benefits from the averaging of the multiple measurements. Nowadays, acquiring up to $80 \%$ overlap images sequences (in both directions) is a common practice among airborne survey companies. In the case of airborne push-broom cameras like the Leica ADS40/80, a side overlap above 50\% provides six well distributed points of views for each point on the ground.

In the computer vision community, several projects aim at producing dense 3D city models from thousands of images found on the Web, which means tens or hundreds of views for a single point.

Up to now, satellite imagery has been a scarce resource and most of space based elevation data have been extracted through single stereoscopic measurements. Nevertheless, the ASTER GDEM and SPOT Ref3D programs have achieved extensive 3D coverages of the earth surface at a nominal 30meter resolution with forward/backward stereoscopic acquisition. Locally, in mountainous areas, the rendering of the Ref3D DEM has been improved, thanks to the triple viewing mode of SPOT5 with its two instruments HRS and HRG.

The new generation of sub-metric earth observation satellite is able to provide more than two images of the same area in a single pass. From our experience with airborne imagery, it should improve greatly the quality and the completeness of a digital elevation model, especially in urban area. On the other hand, satellite imagery is still expensive, and the benefit of multi-view rendering has to be balanced with the extra cost of the acquisition.

One should also notice that, during a one pass acquisition, the satellite is bound to its orbit, which is a single one dimensional path. This is not quite similar to a multi-tracks airborne acquisition with viewing angles evenly distributed around each point on the ground. However, this situation can be partially recreated by using several single-pass stereo pairs acquired from different orbits. Here again, cost matters.

\section{THE STEREO DILEMMA}

The best choice of viewing angles for stereoscopic measurement is always a matter of compromise: A wide stereo angle provides a good geometric accuracy, but the matching of two images points may be difficult, or even impossible if the two points of 
view are very different. In dense urban area, for instance, the simultaneous visibility at the street level is highly dependent on the directions and the incidence angles of the pair of images. In this context, the expected completeness of the elevation surface is easily anticipated from the viewing angles, the estimated buildings heights and streets width.

Regarding the part of the scene visible from both points of view, the performance of the matching, at image level, depends also on how different the two points of view are, but this dependence cannot be theoretically quantified. Many factors are involved in this process, as the matching algorithm, the morphology and the texture of the scene, but, from a statistical point of view, we can say that the matching RMS error $E_{m}$, is an increasing function of the stereoscopic angle $\alpha$ with $E_{m}(0)>0$.

If we assume that the final 3D measurement error is (roughly) $E_{x y z}(\alpha)=E_{m}(\alpha) / \tan \alpha$, an optimum angle value may be found for a given algorithm and a given scene.

If more than two images are available, the previous rule holds for each pairwise matching. If the matching errors are statistically independent from one stereo pair to the other, one can expect an improved accuracy from the merged elevation measure. Unfortunately, the errors are not always independent and, even in this case, the well known $1 / \sqrt{N}$ rule holds only for the RMS value (not for the $90 \%$ error). That is why an experimental approach is required in order to estimate the actual accuracy of each combination of viewing angles.

As far as the robustness and the completeness are concerned, a benefit can be expected from multiple stereo pairs, as long as the additional viewing angles allow sufficient ground visibility and resolution.

This work concentrates on the actual 3D performances obtained from Pleiades images, using various stereoscopic configurations over various landscapes.

\section{OVERVIEW OF THE PROCESSING WORKFLOW}

\subsection{Bundle adjustment}

The attitude biases and drifts of all images are simultaneously estimated, using a set of ground control points and a large number of tie points. The high density of the tie points allows checking the consistency of the data set.

\subsection{Pairwise matching}

Individual stereo pairs are matched separately at the pixel level, using a belief propagation algorithm.

\subsection{D measurements merge}

When several stereo pairs are available, the corresponding DEM are merged with a statistically robust criterion, filtering as much as possible the matching errors.

\section{EXPERIMENTAL RESULTS}

The following experiments were made during a very early stage of the Pleiades validation phase, just after the launch. The interior orientation of the camera was not fully optimised at this time and the results presented here may not reach the ultimate accuracy that will be available when the satellite is officially declared operational. Nevertheless, according to our past experience, these results might be very close to what can be expected in the best conditions from the Pleiades specifications.
Stereoscopic images have been acquired in a single pass over the four following areas:

- Lisboa (2 images)

- Hobart (3 images)

- Montagne Sainte Victoire (2 images)

- Melbourne (17 images)

These sites were previously surveyed with various airborne cameras during the past twelve years. From the oldest of these aerial references, Lisboa and Hobart, we could only extract a few tens of check points. Even if the Melbourne reference is rather old, we could extract a more significant number of points. For these three sites, the check points are concentrated in the dense urban center. The last site, in the south of France is almost fully covered with a recent 5 meter digital terrain model (DTM). A large number of control points were extracted exclusively on bare earth to avoid the discrepancy between the Pleiades DEM and the reference DTM.

The absolute height accuracy of the reference data is estimated between 0.25 and 0.35 meters, depending on the nature of the surface (natural or man-made).

The table below summarizes the geometric configurations of the acquisitions and the final performances measured (in meters) for the best images combination.

\begin{tabular}{|l|r|r|r|r|}
\hline Site & Incidences & Nb. Points & Bias & RMS \\
\hline Lisboa & $-4 / 10$ & 61 & 0.17 & 0.58 \\
\hline Hobart & $-2 / 10 / 15$ & 69 & 0.09 & 0.58 \\
\hline SteVictoire & $-13 / 22$ & 2407 & -0.18 & 0.53 \\
\hline Melbourne & $-49 \ldots+49$ & 295 & 0.27 & 0.49 \\
\hline
\end{tabular}

We will now focus on the two opposite situations represented by Ste Victoire and Melbourne. The first case is a typical acquisition configuration over an open landscape, with a wide stereo angle aiming at maximizing the geometric accuracy without too much care about visibility limitation. The second case is a nice test bed for experimentation and quantification of the "stereo dilemma" in a dense urban context.

\subsection{Montagne Sainte-Victoire}

A single pair was acquired with a rather wide stereo angle, providing a good accuracy on moderate reliefs. The situation is more difficult on the south face of the mountain, with a concentration of very steep slopes. Locally, we noticed losses of resolution, due to a line of sight nearly tangent to the terrain.

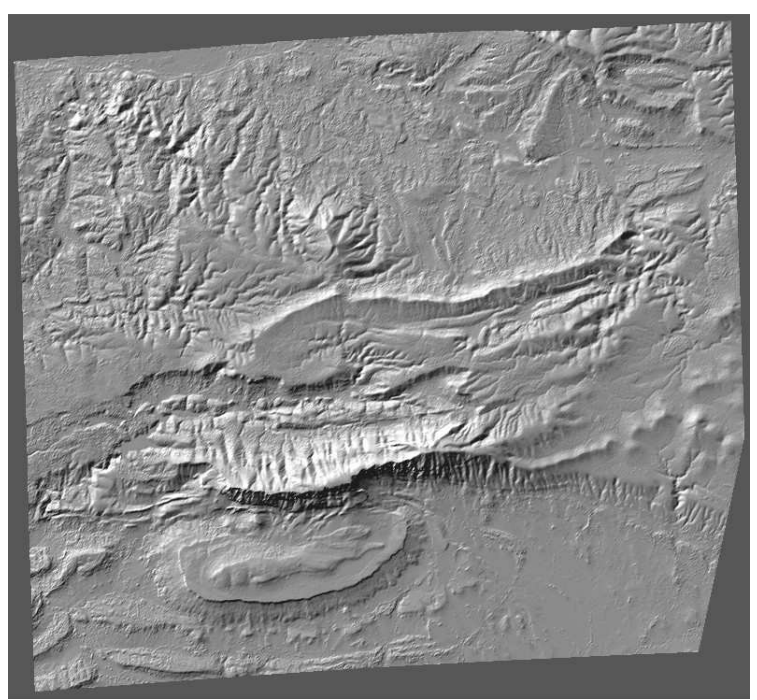

Figure 1 Montagne Sainte Victoire DEM 
A slight distortion of the internal model of the camera can also be seen in the spatial distribution of the height errors (Figure 2).

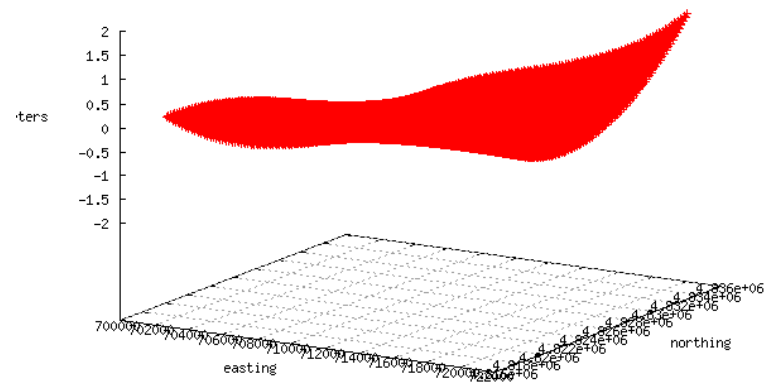

Figure 2 Polynomial approximation of the height errors

In spite of these sparse artefacts, the overall quality of the elevation surface is not very far from what we usually extract from airborne imagery and we can even expect an improvement of the accuracy with a better knowledge of the camera geometry after the completion of the commissioning phase of the satellite.

\subsection{Melbourne}

A sequence of 17 images was acquired from $-49.6^{\circ}$ to $48.7^{\circ}$ along the track, evenly distributed every 5 to 7 degrees.
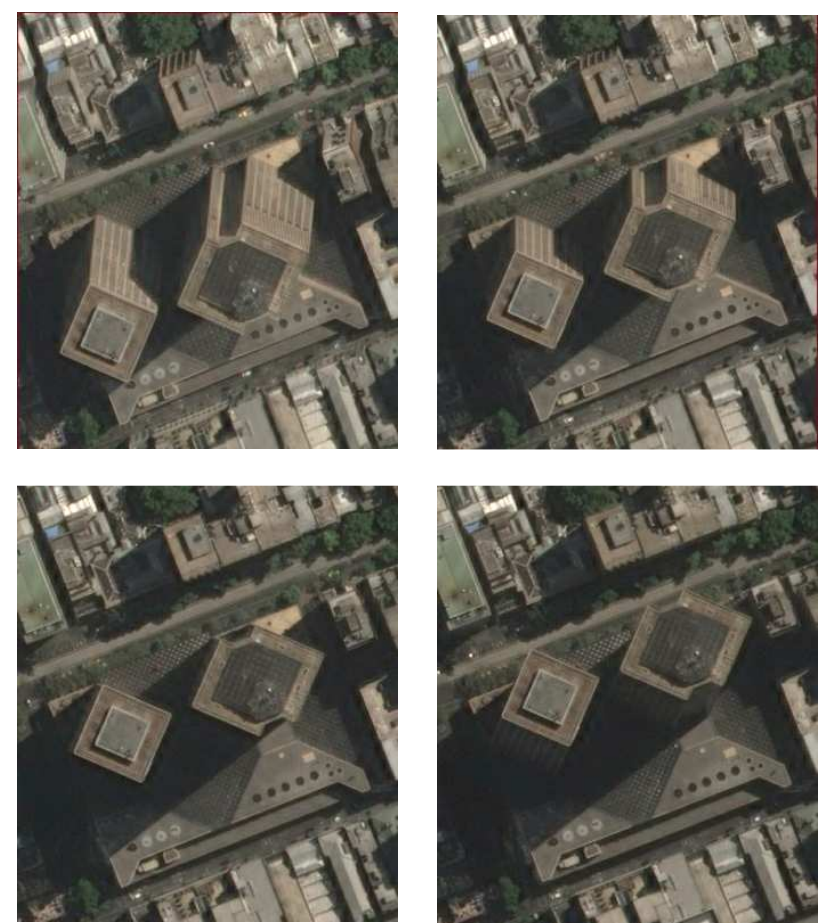

Figure 3 Melbourne sequence samples

A set of 295 check points have been manually chosen on the reference DEM, partly on the ground and partly on the buildings roofs (Figure 4), checking that no change occurs between the airborne survey and the Pleiades acquisitions (the west part, near the stadium is obsolete in the reference data).

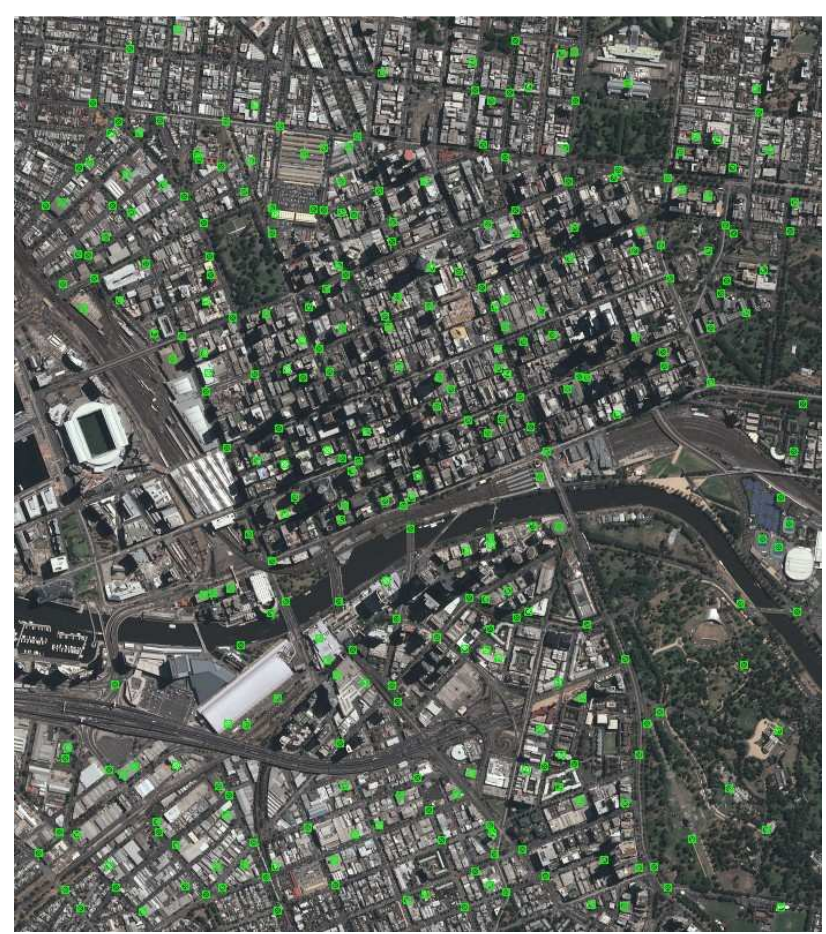

Figure 4 Melbourne ground control points

All pairs with a stereo angle of (approximately) $15^{\circ}$ have been processed. In a second time, we processed also the pairs with a $10^{\circ}$ stereo angle, from $-21.7^{\circ}$ to $20.7^{\circ}$.

The tables below summarize the individual performance of each stereo pair, in terms of completeness and accuracy.

\begin{tabular}{|c|r|r|r|r|}
\hline Incidences & Nb. Points & Bias & RMS & LE90 \\
\hline$-49.6 /-28.4$ & 141 & 0.44 & 1.11 & 1.24 \\
\hline$-42.3 /-21.7$ & 207 & 0.05 & 0.73 & 1.14 \\
\hline$-35.2 /-16.0$ & 244 & 0.18 & 0.83 & 0.95 \\
\hline$-28.4 /-11.0$ & 264 & 0.28 & 0.69 & 1.01 \\
\hline$-21.7 /-5.8$ & 278 & 0.45 & 0.87 & 1.24 \\
\hline$-16.0 /-0.3$ & 285 & 0.26 & 0.66 & 1.08 \\
\hline$-11.0 / 5.1$ & 291 & 0.37 & 0.75 & 1.12 \\
\hline$-5.8 / 10.4$ & 285 & 0.23 & 0.73 & 0.99 \\
\hline$-0.3 / 15.8$ & 277 & 0.24 & 0.58 & 0.97 \\
\hline $5.1 / 20.7$ & 271 & 0.29 & 0.96 & 0.99 \\
\hline $10.4 / 27.4$ & 259 & 0.22 & 0.65 & 1.01 \\
\hline $15.8 / 34.3$ & 238 & 0.22 & 0.72 & 1.08 \\
\hline $20.7 / 41.4$ & 220 & 0.33 & 0.81 & 1.06 \\
\hline $27.4 / 48.7$ & 203 & 0.26 & 0.90 & 1.14 \\
\hline
\end{tabular}

Table 1 Wide angle stereo pairs (base/height $=0.26-0.36$ )

\begin{tabular}{|c|r|r|r|r|}
\hline Incidences & Nb. Points & Bias & RMS & LE90 \\
\hline$-21.7 /-11.0$ & 270 & 0.52 & 0.93 & 1.52 \\
\hline$-16.0 /-5.8$ & 277 & 0.51 & 0.97 & 1.52 \\
\hline$-11.0 /-0.3$ & 289 & 0.49 & 0.92 & 1.38 \\
\hline$-5.8 / 5.1$ & 291 & 0.42 & 0.78 & 1.24 \\
\hline$-0.3 / 10.4$ & 292 & 0.39 & 0.89 & 1.32 \\
\hline $5.1 / 15.8$ & 278 & 0.38 & 1.01 & 1.34 \\
\hline $10.4 / 20.7$ & 273 & 0.51 & 1.17 & 1.47 \\
\hline
\end{tabular}

Table 2 Narrow angle stereo pairs (base/height $=0.18$ )

Those results underline clearly two phenomena:

- The completeness of the measures is highly dependent on the incidence angles (Figure 5). 
- A moderate stereo angle is definitely more efficient than a narrow angle in term of accuracy, with no difference, in term of completeness.

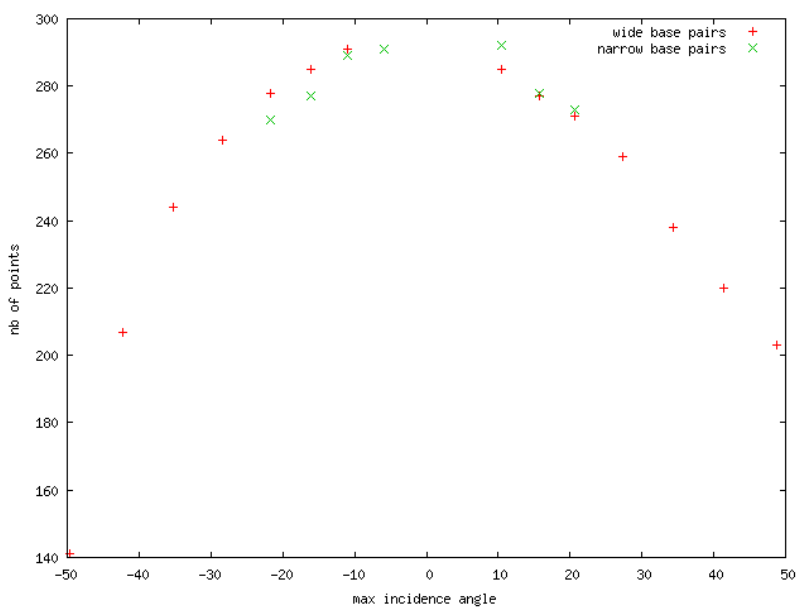

Figure 5 Completeness versus incidence angles

We merged different combination of stereo measurements, using 3, 7 and 9 pairs. A first set is composed of wide angle stereo pairs, a second set is made from narrow angle stereo pairs. Our main concern is the evolution of the accuracy when the individual measures are aggregated. A decreasing RMS error would suggest the statistical independence of the different measures. The results below show that we are far from this situation. Aggregating 2 stereo pairs (3 images) improves clearly RMS and LE90 performances, but adding more images brings only a slight decrease in both values. In the case of narrow stereo angles, the aggregation benefit for 3 images is higher, but not sufficient to compete with the performance obtained from wide stereo angle measures.

\begin{tabular}{|c|c|r|r|r|r|}
\hline Incidences & Stereo & Images & Bias & RMS & LE90 \\
\hline$-16.0 . .15 .8$ & Wide & 3 & 0.24 & 0.52 & 0.84 \\
\hline$-11.0 . .10 .4$ & Narrow & 3 & 0.37 & 0.68 & 1.15 \\
\hline$-21.7 . .20 .7$ & Wide & 7 & 0.25 & 0.49 & 0.80 \\
\hline$-16.0 . .15 .8$ & Narrow & 7 & 0.37 & 0.61 & 1.00 \\
\hline$-28.4 . .27 .4$ & Wide & 9 & 0.27 & 0.49 & 0.78 \\
\hline$-21.7 . .20 .7$ & Narrow & 9 & 0.38 & 0.62 & 0.96 \\
\hline
\end{tabular}

Table 3 Multi stereo pairs

The benefit of the aggregation for the completeness is more obvious. For all combinations, the 295 check points are well defined in the aggregated DEM. This has to be also related to the perfect symmetry of the acquisition around a nadir image: This configuration minimises the hidden parts in a dense urban environment. Obviously, this condition cannot be met everywhere in the world because helio-synchronous satellites fly only along a finite set of orbits, which are generally not at the exact zenith of the area of interest.

\section{CONCLUSION}

We have presented a systematic exploration of the stereoscopic capabilities of Pleiades imagery for the extraction of digital elevation models in various environments. The agility of the satellite has risen questions about what is feasible, and what is efficient, regarding 3D mapping from multi-angle images sequences. As expected, the answer depends on the context. In an open landscape situation, without severe occlusions, a single stereo pair should provide a nearly optimal result with a fairly wide stereo angle. In urban area, the main profit of multi-angle

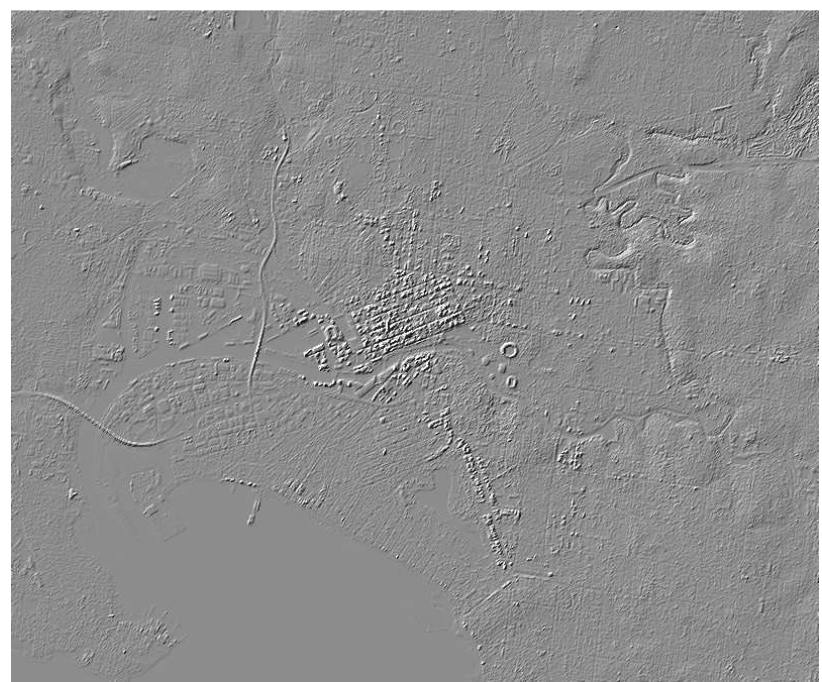

Figure 6 Melbourne DEM (7 images)
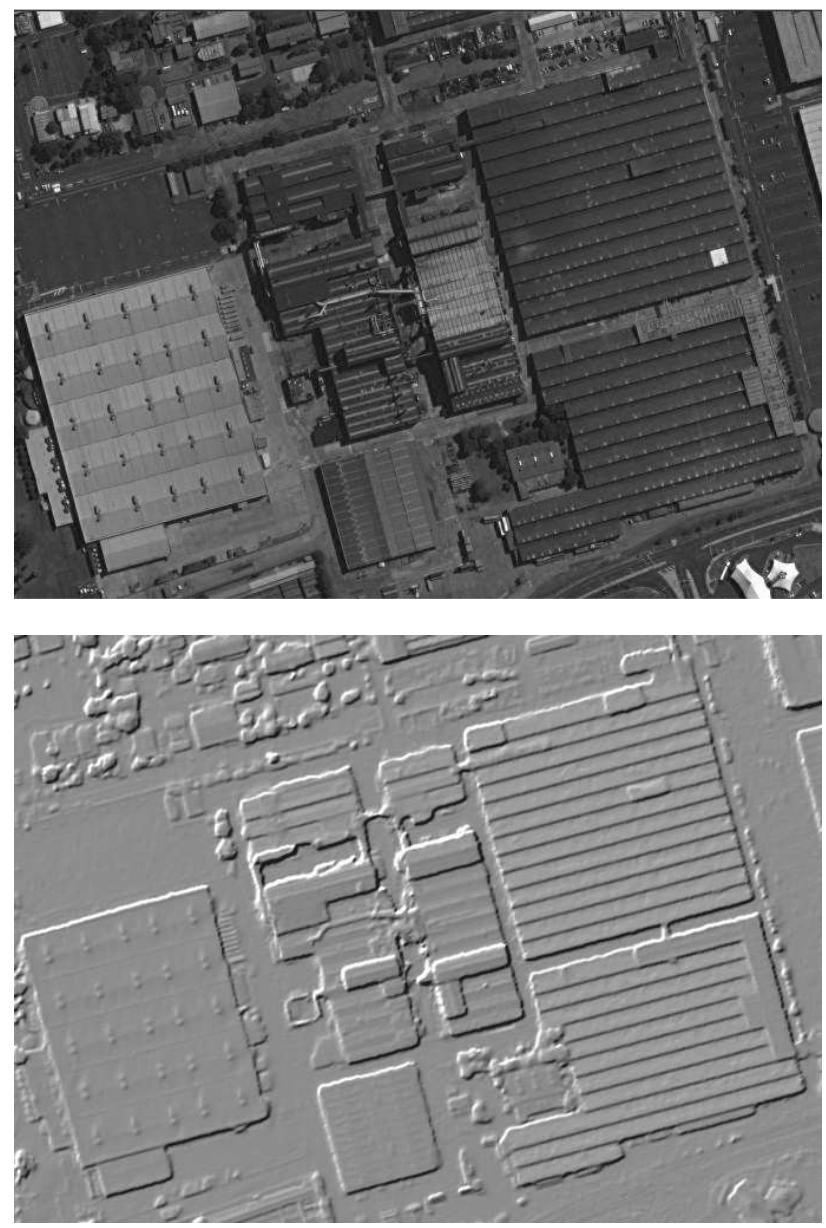

Figure 7 Melbourne DEM (detail)

images sequences is the reduction of occlusions behind buildings. A symmetric configuration with two oblique views on each side of a nadir image seems to be a good compromise in this context. Additional images acquired on the same pass would not increase the scene visibility and bring no significant accuracy improvement. The stereoscopic angle has to be consistent with the scene morphology to avoid occlusions but we did not get convincing results from narrow angles configuration. 
Of course, these conclusions are related to a particular processing method of pair wise matching. We checked that a standard method based on correlation leads to the same observations but other approaches exist, matching all images simultaneously, for instance. The Melbourne sequence could be an appropriate benchmark for a wide spectrum evaluation of 3D reconstruction techniques, making sure that all valuable information acquired by the satellite is correctly handled.

\section{REFERENCES}

Haala, N. (2009) Comeback of Digital Image Matching, Photogrammetric Week 2009, Wichmann Verlag pp. 289 - 301.

Jan-Michael Frahm, Pierre Fite-Georgel, David Gallup, Tim Johnson, Rahul Raguram, Changchang Wu, Yi-Hung Jen, Enrique Dunn, Brian Clipp, Svetlana Lazebnik, and Marc Pollefeys. 2010. Building Rome on a cloudless day. In Proceedings of the 11th European conference on Computer vision: Part IV (ECCV'10), Kostas Daniilidis, Petros Maragos, and Nikos Paragios (Eds.). Springer-Verlag, Berlin, Heidelberg, 368-381.

http://www.astrium-geo.com/en/2788-reference3d-yourgeographic-reference-system

http://www.gdem.aster.ersdac.or.jp/ 